\title{
Using Google Dengue Trends to Estimate Climate Effects in Mexico
}

\author{
Rebecca T. Gluskin*1, Mauricio Santillana ${ }^{2}$ and John S. Brownstein ${ }^{1,3}$ \\ 'Boston Children's Hospital, Boston, MA, USA; ${ }^{2}$ Harvard School of Engineering and Applied Sciences, Cambridge, MA, USA; \\ ${ }^{3}$ Department of Pediatrics, Harvard Medical School, Boston, MA, USA
}

\section{Objective}

To evaluate the association between Dengue Fever (DF) and climate in Mexico with real-time data from Google Dengue Trends (GDT) and climate data from NASA Earth observing systems.

\section{Introduction}

The incidence of dengue fever (DF) has increased 30 fold between 1960 and 2010 [1]. The literature suggests that temperature plays a major role in the life cycle of the mosquito vector and in turn, the timing of DF outbreaks [2]. We use real-time data from GDT and real-time temperature estimates from NASA Earth observing systems to examine the relationship between dengue and climate in $17 \mathrm{Mex}$ ican states from 2003-2011. For the majority of states, we predict that a warming climate will increase the number of days the minimum temperature is within the risk range for dengue.

\section{Methods}

The GDT estimates are derived from internet search queries and use similar methods as those developed for Google Flu Trends [3]. To validate GDT data, we ran a correlation between GDT and dengue data from the Mexican Secretariat of Health (2003-2010). To analyze the relationship between GDT and varying lags of temperature, we constructed a time series meta-analysis. The mean, max and min of temperature were tested at lags $0-12$ weeks using data from the Modern Era Retrospective-Analysis for Research and Applications. Finally, we built a binomial model to identify the minimum $5^{\circ} \mathrm{C}$ temperature range associated with a $50 \%$ or higher Dengue activity threshold as predicted by GDT.

\section{Results}

The time series plot of GDT data and the Mexican Secretariat of Health data (2003- 2010) (Figure 1) produced a correlation coefficient of 0.87 . The time series meta-analysis results for 17 states showed an increase in minimum temperature at lag week 8 had the greatest odds of dengue incidence, 1.12 Odds Ratio (1.09-1.16, 95\% Confidence Interval). The comparison of dengue activity above $50 \%$ in each state to the minimum temperature at lag week 8 showed 14/17 states had an association with warmest 5 degrees of the minimum temperature range. The state of Sonora was the only state to show an association between dengue and the coldest 5 degrees of the minimum temperature range.

\section{Conclusions}

Overall, the incidence data from the Mexican Secretariat of Health showed a close correlation with the GDT data. The meta-analysis indicates that an increase in the minimum temperature at lag week 8 is associated with an increased dengue risk. This is consistent with the Colon-Gonzales et al. Mexico study which also found a strong association with the 8 week lag of increasing minimum temperature [4]. The results from this binomial regression show, for the majority of states, the warmest 5 degree range for the minimum temperature had the greatest association with dengue activity 8 weeks later. Inevitably, several other factors contribute to dengue risk which we are unable to include in this model [5]. IPCC climate change predictions suggest a $4^{\circ} \mathrm{C}$ increase in Mexico. Under such scenario, we predict an increase in the number of days the minimum temperature falls within the range associated with DF risk.

Google Dengue Trends vs. Secretariat of Health Data, Mexico

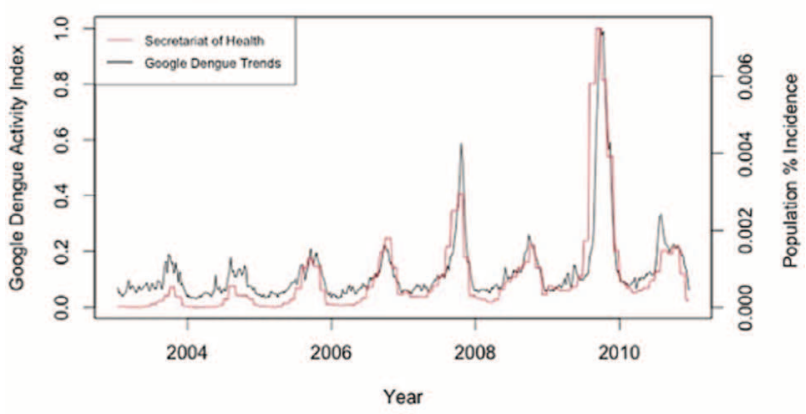

Figure 1. Time Series Correlation: Google Dengue Trends vs. Secretariat of Health, Mexico 2003-2010

\section{Keywords}

Time Series; Mexico; Google Dengue Trends; Climate Change; Meta-analysis

\section{Acknowledgments}

Funded by the NIH Grant \# 1R01LM010812-01 and the Applied Public Health Informatics Fellowship Program administered by CSTE and funded by the Centers for Disease Control and Prevention (CDC) Cooperative Agreement 3U38HM000414-04W1.

\section{References}

1.(WHO), W.H.O., Dengue. 2010.

2.Yang, H.M., et al., Assessing the effects of temperature on the population of Aedes aegypti, the vector of dengue. Epidemiol Infect, 2009. 137(8): p. 1188-202

3.Chan, E.H., et al., Using web search query data to monitor dengue epidemics: a new model for neglected tropical disease surveillance. PLoS Negl Trop Dis, 2011. 5(5): p. e1206.

4.Colon-Gonzalez, F.J., I.R. Lake, and G. Bentham, Climate variability and dengue fever in warm and humid Mexico. Am J Trop Med Hyg, 2011. 84(5): p. 757-63.

5.Thai, K.T. and K.L. Anders, The role of climate variability and change in the transmission dynamics and geographic distribution of dengue. Exp Biol Med (Maywood), 2011. 236(8): p. 944-54.

*Rebecca T. Gluskin

E-mail: rgluskin@health.nyc.gov 